\title{
Osimertinib and dihydroartemisinin: a novel drug combination targeting head and neck squamous cell carcinoma
}

\author{
Imane Chaib ${ }^{1}$, Xueting Cai ${ }^{2,3}$, David Llige ${ }^{1}$, Mariacarmela Santarpia ${ }^{4}$, Eloisa Jantus-Lewintre ${ }^{5,6,7}$, \\ Martyna Filipska ${ }^{1}$, Carlos Pedraz ${ }^{1}$, Jean Cui ${ }^{8}$, Jie Yang $^{2}$, Jing Miao ${ }^{2}$, Rongwei Sun ${ }^{2}$, \\ Jillian Wilhelmina Paulina Bracht ${ }^{9}$, Masaoki Ito ${ }^{9}$, Jordi Codony-Servat ${ }^{9}$, Niki Karachaliou ${ }^{10}$, \\ Andrés Aguilar ${ }^{10}$, Rafael Rosell ${ }^{1,9,10,11}$, Peng $\mathbf{C a o}^{2,3,12}$
}

${ }^{1}$ Institut d'Investigació en Ciències de la Salut Germans Trias i Pujol, Badalona, Spain; ${ }^{2}$ Affiliated Hospital of Integrated Traditional Chinese and Western Medicine, Nanjing University of Chinese Medicine, Nanjing 210028, China; ${ }^{3}$ College of Pharmacy, Nanjing University of Chinese Medicine, Nanjing 210023, China; ${ }^{4}$ Medical Oncology Unit, Department of Human Pathology “G. Barresi”, University of Messina, Messina, Italy; ${ }^{5}$ Molecular Oncology Laboratory, Fundación Hospital General Universitario de Valencia, Valencia, Spain; ${ }^{6}$ CIBERONC, Valencia, Spain; ${ }^{7}$ Department of Biotechnology, Universitat Politècnica de València, Valencia, Spain; ${ }^{8} \mathrm{TP}$ Therapeutics, Inc., San Diego, CA, USA; ${ }^{9}$ Pangaea Oncology, Laboratory of Molecular Biology, Quirón-Dexeus University Institute, Barcelona, Spain; ${ }^{10}$ Instituto Oncológico Dr. Rosell (IOR),

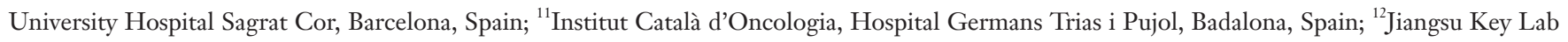
of Cancer Biomarkers, Prevention and Treatment, Jiangsu Collaborative Innovation Center for Cancer Personalized Medicine, School of Public Health, Nanjing Medical University, Nanjing 211166, China

Contributions: (I) Conception and design: R Rosell; (II) Administrative support: None; (III) Provision of study materials or patients: None; (IV) Collection and assembly of data: I Chaib, X Cai, J Yang, J Miao, R Sun, D Lligé; (V) Data analysis and interpretation: None; (VI) Manuscript writing: All authors; (VII) Final approval of manuscript: All authors.

Correspondence to: Rafael Rosell, MD, PhD. Institut d’Investigació en Ciències de la Salut Germans Trias i Pujol, Badalona 08916, Spain. Email: rrosell@iconcologia.net; Peng Cao, PhD. Affiliated Hospital of Integrated Traditional Chinese and Western Medicine, Nanjing University of Chinese Medicine, Nanjing 210028, China. Email: cao_peng@njucm.edu.cn.

Background: Recurrent and metastatic head and neck squamous cell carcinoma (HNSCC) has a dismal prognosis with limited progression-free survival and overall survival, even when treated with different combinations of chemotherapy, targeted therapies and immunotherapy. We explored in vitro and in vivo the effect of the epidermal growth factor receptor (EGFR) inhibitor, osimertinib, alone and in combination with dihydroartemisinin (DHA) in HNSCC.

Methods: The combination of osimertinib with DHA was tested in the FaDu and CAL27 HNSCC cell lines. Tumor cell proliferation assays were conducted in cultured cells and mouse xenografts. Western blotting analysis of related signal pathways was performed to investigate the molecular mechanisms of the inhibitory effect of DHA and the combination. Other compounds, which inhibit signal transducer and activator of transcription 3 (STAT3), Src-family kinases (SFKs), sphingosine kinase 1 (SPHK1), or the receptor tyrosine kinase (RTK) AXL were also combined with osimertinib in vitro.

Results: Osimertinib exerted synergistic cytotoxicity toward FaDu and CAL27 HNSCC cells when combined with DHA. DHA reversed the osimertinib-induced STAT3 and Src phosphorylation. The double combination inhibited AXL expression. The anticancer potential of osimertinib plus DHA combination was validated in vivo on FaDu and CAL27 xenografts in mice without notable side effects.

Conclusions: The results illustrate that the combinatory therapy of osimertinib and DHA, as a repurposing anticancer drug, could be a novel therapeutic strategy for recurrent and/or metastatic HNSCC patients. The findings strongly indicate that a clinical trial is warranted to confirm the benefit of the combination.

Keywords: Head and neck; dihydroartemisinin (DHA); osimertinib; repurposing drug; drug combination

Submitted Jul 05, 2019. Accepted for publication Sep 23, 2019.

doi: $10.21037 /$ atm.2019.10.80

View this article at: http://dx.doi.org/10.21037/atm.2019.10.80 


\section{Introduction}

Recurrent or metastatic head and neck squamous cell carcinoma (HNSCC) has poor prognosis and limited overall survival with chemotherapy and/or the epidermal growth factor receptor (EGFR) antibody, cetuximab (1). Activation of EGFR signaling in HNSCC occurs through multiple mechanisms, including overexpression of EGFR and its ligands, EGFR gene amplification or mutations, and EGFR transactivation by other receptor tyrosine kinases (RTKs) like AXL (2,3). The molecular mechanisms underlying the acquired resistance to EGFR inhibitors, in other tumor types, like lung cancer, have been widely explored. The activation of AXL, Janus kinase 2 (JAK2)/ signal transducer and activator of transcription 3 (STAT3) and phosphatidylinositol-3 kinase (PI3K)/AKT signaling, have been demonstrated as key signaling pathways in the development of resistance to EGFR inhibitors (4-6).

AXL is overexpressed in non-small-cell lung cancer (NSCLC) and HNSCC tumors with intrinsic resistance to the anti-EGFR monoclonal antibody, cetuximab (7). AXL upregulation was also observed in erlotinib-resistant HNSCC cells responding to an AXL inhibitor (R428). Moreover, elevated AXL messenger ribonucleic acid (mRNA) levels were linked to poor survival among 302 HNSCC patients (8). AXL overexpression is more common in triple negative breast cancer (TNBC), HNSCC, and NSCLC (9). In HNSCC patients with activating mutations of the p110 $\alpha$ subunit of PI3K (PIK3CA) treated with a PI3 K $\alpha$ inhibitor (BYL719), AXL appeared as mechanism of resistance (10). Median progression-free survival was 4.6 months for patients receiving buparlisib (BKM 120), a pan-PI3K inhibitor (11). The treatment of TNBC and NSCLC cell lines with the dual inhibitor of AXL and MET (BMS777607) resulted in more MET, AXL, as well as Src phosphorylation, compared to untreated cells, while the Src inhibitor, dasatinib, prevented the activation of MET and AXL (12). We also noted AXL activation in EGFRmutation positive cell lines, that was abrogated by either silencing YES associated protein 1 (YAP1), Src, YES, and LYN (Src associated proteins), or by treating the cells with the Src/focal adhesion kinase (FAK)/JAK2 inhibitor (TPX0005, also known as repotrectinib). The combination of osimertinib and TPX-0005 was highly synergistic in vitro and in vivo (4).

Apart from EGFR kinases, osimertinib also shows significant activity in other kinases, such as, ERBB2, ERBB4 and B-lymphoid kinase (BLK) (13), and increases the intracellular accumulation of doxorubicin by inhibiting the efflux function of ATP-binding cassette (ABC) transporters in $\mathrm{ABCB} 1$ - or ABCG2 - overexpressing cells (14). The transcriptional inhibitor, E2F7, is mislocalized to the cytoplasm in more than $80 \%$ of human HNSCCs, with activation of sphingosine kinase 1 (SPHK1), causing anthracycline resistance (15). Cetuximab-resistant HNSCC cells show activation of bromodomain-containing protein-4 (BRD4), that regulates the transcription of MET and AXL (16). An interaction of YAP1/transcriptional coactivator with PDZ-binding motif (TAZ) with BRD4 leads to increased transcription of a large set of genes, including AXL and the immune checkpoint programmed death-ligand 1 (PD-L1) (17). The median progressionfree survival to nivolumab, an anti-PD-1 monoclonal antibody, was 2 months in recurrent HNSCC (18). Dihydroartemisinin (DHA), a front-line antimalarial herb compound, is a semisynthetic derivative of artemisinin that has shown promising anticancer activity in vitro and in vivo with low toxicity to normal cells (19). DHA inhibits the activation of STAT3 and increases the anti-proliferative effect of cisplatin in HNSCC cell lines (20).

In the current study, instead of cetuximab, we opted for osimertinib, an irreversible EGFR tyrosine kinase inhibitor (TKI) in HNSCC models. We used a 4-pronged approach of osimertinib with DHA, an AXL inhibitor (R428), a Src/ FAK/JAK2 inhibitor (TPX-0005), or a SPHK1 inhibitor (BML258).

\section{Methods}

\section{Cell lines and reagents}

The human hypopharyngeal carcinoma cell line, FaDu and the human tongue squamous cell carcinoma cell line, CAL27 were purchased from the American Type Culture Collection (ATCC) in October of 2017-ATCC generates buman STR profiles by simultaneously amplifying multiple STR loci and amelogenin (for gender determination) using the Promega PowerPlex ${ }^{\circledR}$ System ${ }^{2}$. Amplicons are separated by capillary electrophoresis and analyzed using GeneMapper ${ }^{\circledR}$ Software from Thermo Fisher Scientific (https://www. lgcstandards-atcc.org/Products/Cells \% 20 and \% 20 Microorganisms/Testing\%20and\%20Characterization/ STR\%20Profiling\%20Analysis.aspx?geo_country=es). The cell lines were cultured according to the manufacturer's online instructions. Specifically, FaDu and CAL27 cells were maintained in Eagle's Minimum Essential Medium 
(EMEM) and Dulbecco's Modified Eagle Medium nutrient mixture F-12 (DMEM-F12), respectively. Mediums were supplemented with $1 \%$ penicillin/streptomycin/glutamine (Gibco; Thermo Fisher Scientific, Inc., Waltham, MA, USA) and $10 \%$ fetal bovine serum (FBS; Gibco; Thermo Fisher Scientific, Inc.) in a $5 \% \mathrm{CO} 237^{\circ} \mathrm{C}$ cell culture incubator and routinely evaluated for mycoplasma contamination. Osimertinib, DHA, R428, and BML258 were purchased from Selleck Chemicals (Houston, TX, USA). TPX-0005 was kindly provided by TP Therapeutics, Inc (San Diego, CA, USA) under a material transfer agreement. Drugs were prepared in dimethyl sulfoxide (DMSO) at a concentration of $10-100 \mathrm{mmol} / \mathrm{L}$ stock solutions and stored at $-20{ }^{\circ} \mathrm{C}$. Further dilutions were made in culture medium to final concentration before use.

The phospho-specific YAP1 (Tyr357) and the paxillin (phospho-specific and total) antibodies were purchased from Abcam (Cambridge, MA, USA). The B-actin antibody was from Sigma Aldrich (St Louis, MO, USA). The rest of phospho-specific and total antibodies used in this study were purchased from Cell Signaling Technology (Beverly, MA, USA). The secondary antibodies Amersham ECLanti-rabbit IgG horseradish peroxidase-linked speciesspecific whole antibody, and Amersham ECL-anti-mouse IgG peroxidase-linked whole antibody were purchased from GE Healthcare UK limited (Buckinghamshire, UK).

\section{Cell viability in vitro}

Cells were seeded on 96-well plates at $3.5 \times 10^{3}$ and incubated for 24 hours. Next, the cells were treated for 72 hours with serial dilutions of the drugs administrated at doses typically corresponding to $1 / 8,1 / 4,1 / 2,5 / 8,3 / 4,7 / 8,1,1.5$ and 3 of the individual half maximal inhibitory concentration (IC50) values. After incubation, freshly prepared MTT (tetrazoliumbased semiautomated colorimetric 3(4,5-dimethylthiazol2-yl)-2,5-diphenyltetrazolium bromide) reagent (Sigma Aldrich, St Louis, MO, USA) $(0.5 \mathrm{mg} / \mathrm{mL})$ was added to the medium in the wells for 2 hours at $37^{\circ} \mathrm{C}$. Formazan crystals in viable cells were solubilized with $100 \mu \mathrm{L}$ DMSO. The amount of formazan formed was determined by measuring the optical density (absorbance) at $550 \mathrm{~nm}$ using a Varioskan Flash 96-well microplate reader (Thermo Fisher Scientific, Massachusetts, USA). The mean absorbance of four wells in the indicated groups was used to calculate the cell viability as follows: cell viability = $\left(A_{\text {treatment }}-A_{\text {blank }}\right) /\left(A_{\text {control }}-A_{\text {blank }}\right)$ (where, $A=$ absorbance $)$. Data of combined drug effects were analyzed by the Chou-
Talalay method (21). Combination index values $<1,=1$ and $>1$ indicated synergism, additive effect and antagonism, respectively, as previously described $(4,5)$.

For the colony formation assay, cells were plated in sixwell plates at 1,000 cells/well in medium, 10\% FBS. The cells were cultured for 24 hours and the media were then replaced with medium, 1\% FBS with or without inhibitors. After 72 hours the media were removed and replaced with fresh media without inhibitors for a total of 10 days. At the end of the experiment, the media were removed, and the cells were washed with phosphate-buffered saline (PBS). The colonies were fixed and stained simultaneously with $0.5 \%$ crystal violet in $10 \%$ of ethanol for $15 \mathrm{~min}$. The stain was aspirated, and the wells washed with deionized water until the background was clear. The wells were then photographed and counted using Image $\mathrm{J}$ software.

\section{Western blotting and gene expression analyses}

Cells were washed with cold PBS and re-suspended in icecold radio-immunoprecipitation assay (RIPA) buffer (20 mM Tris- hydrochloric acid in $\mathrm{pH} 7.5,1 \%$ Nonidet P-40, $0.5 \%$ sodium deoxycholate, $150 \mathrm{mM}$ sodium chloride, $1 \mathrm{mM}$ ethylenediaminetetraacetic acid, $1 \mathrm{mM}$ EGTA, $2.5 \mathrm{mM}$ sodium pyrophosphate, $1 \mathrm{mM}$ beta-glycerophosphate and $1 \mathrm{mM}$ sodium vanadate) containing protease inhibitor mixture. Following cell lysis and centrifugation at $14,000 \times \mathrm{rpm}$ for $10 \mathrm{~min}$ at $4{ }^{\circ} \mathrm{C}$, the resulting supernatant was collected as the total cell lysate. Briefly, equivalent amounts of proteins were separated by $8 \%$ or $10 \%$ SDSPAGE (Life Technologies, Carlsbad, CA, USA), and then transferred onto PVDF membranes (Bio-Rad laboratories Inc., Hercules, CA, USA). Membranes were blocked in Odyssey blocking buffer (Li-Cor Biosciences, Lincoln, NE, USA) and incubated with appropriate primary antibodies overnight. Membranes were incubated with peroxidase conjugated secondary antibodies for $1 \mathrm{~h}$ at room temperature. Quimioluminiscence bands were detected with Bio-Rad ChemiDoc MP Imaging System.

Total RNA was extracted from harvested cells using TRIzol reagent (Thermo Fisher Scientific, Waltham, MA, USA). The mRNA was reverse transcribed into cDNA using the M-MLV Retro-Transcription Kit (Thermo fisher). Quantitative real-time PCR was conducted on a 7500 Real-Time PCR System (Applied Biosystems, Carlsbad, CA, USA) using TaqMan detection protocol as previously described $(4,5)$. Expression levels were normalized to B-actin mRNA expression. Data were calculated according to the 
comparative $\Delta \Delta \mathrm{Ct}$ method. Commercial RNA controls were used as calibrators (Liver and Lung; Stratagene, La Jolla, CA, USA). For each cell line, three independent experiments were performed. The primer and probe sets were designed using Primer Express 3.0 Software (Applied Biosystems) according to their Ref Seq (http://www.ncbi. nlm.nih.gov/LocusLink). Specifically, sequences of the primer sets and the probes for amplification of the genes examined in the study were as follows: AXL, forward primer, 5'-CAGCGCAGCCTGCATGT-3', reverse primer, 5'-GCGTTATGGGCTTCGCAG-3', probe 5'-FAM CAGGGCTGAACAAGAC-MGB 3'; MET, forward primer, 5'-TCACCATAGCTAATCTTGGGACATC-3', reverse primer, 5'-GTTGATGGTCCTGATCGAGAAAC-3', probe 5'-FAM TCGCTTCATGCAGGTTGM G B 3 '; C D C P 1, forward primer, 5'-AGCTGCCAGAGTCCTGAGAATC-3', reverse primer, 5'-TGGGCCTGACATACAGTCAATATT-3', probe 5'FAM ACTTTGTCATAGAGATCCA-MGB 3'; GPX4, Hs00989766 assay (Applied Biosystems); $\beta$-actin, forward primer, 5'-TGAGCGCGGCTACAGCTT-3', reverse primer, 5'-TCCTTAATGTCACGCACGATTT-3', probe 5'-FAM ACCACCA CGGCCGAGCGGTA-MRA 3'.

\section{Xenograft studies}

All procedures were based on Guide for Care and Use of Laboratory Animals of National Institutes of Health and approved by Institutional Animal Care and Use Committee of Jiangsu Province Academy of Traditional Chinese Medicine (SYXK 2016-0018). Nude mice (16 \pm 2 g) were obtained from Changzhou Cavens Lab Animal Company (Changzhou, Jiangsu, China). All animals were maintained in a clean facility in Jiangsu Province Academy of Traditional Chinese Medicine (Nanjing, Jiangsu, China). Mice were kept in individually ventilated cages (IVC) cages (5 per cage) with free access of food and water, at $20{ }^{\circ} \mathrm{C}$ and $50 \% \pm 20 \%$ relative humidity under a $12 / 12 \mathrm{~h}$ light/dark cycles and pathogen free conditions. A suspension of $4 \times 10^{6} \mathrm{FaDu}$ or CAL27 cells resuspended in PBS, mixed 1:1 with Corning Matrigel Basement Membrane Matrix, was injected subcutaneously into the right flank of the mice. Mice with tumors of approximately $100 \mathrm{~mm}^{3}$ were randomized into five groups each containing five mice. Mice were treated once a day orally for one month with $0.5 \%$ carboxymethyl cellulose sodium salt (vehicle), osimertinib $5 \mathrm{mg} / \mathrm{kg}$, DHA $50 \mathrm{mg} / \mathrm{kg}$, or osimertinib plus DHA. The body weight and tumor size were measured once every three days. Tumor volume was calculated as follows: length $\times\left(\right.$ width $\left.^{2}\right) \times 0.5$. At the end of the experiments, all animals were sacrificed, and the tumors were excised and weighed.

\section{Statistical analysis}

Statistical analysis was performed using the GraphPad Prism 7 software (GraphPad Software, Inc, San Diego, CA, USA). The statistical significance was analyzed by one- sided or two-sided tests. $\mathrm{P}$ values less than .05 were considered statistically significant.

\section{Results}

\section{Sensitivity of HNSCC Cells to Osimertinib}

We first tested the treatment effect of osimertinib in HNSCC cells. Specifically, the EGFR-overexpressing $\mathrm{FaDu}$ and CAL27 HNSCC cell lines (22) were treated with osimertinib. Osimertinib inhibited HNSCC cell growth in a dosedependent manner (Figure S1). The half maximal inhibitory concentration (IC50) of osimertinib was $0.2 \pm 0.1 \mu \mathrm{M}$ in both cell lines. These data indicate that osimertinib has an antiproliferative effect on the growth of HNSCC cells in vitro.

\section{Synergistic effect of osimertinib and $\mathrm{DH} A$}

We then tested whether the effect of osimertinib on HNSCC cells can be enhanced when it is rationally combined with compounds that target STAT3, AXL, Src, or SPHK1. The IC50s of each of these compounds in $\mathrm{FaDu}$ and CAL27 cells ranged from 1 to $20 \mu \mathrm{M}$ and are shown in Table S1. The combination of osimertinib with DHA, R428, TPX-0005, or BML-258 exerted synergistic cytotoxicity towards the two HNSCC cell lines with combination index values of less than 1 (Figure 1). Osimertinib combined with DHA distinguished from other combinations used in our study, with combination index values of 0.468 and 0.593 in $\mathrm{FaDu}$ and CAL27 cells, respectively (Figure 1). The FaDu and CAL27 colony formation capacity was significantly reduced with osimertinib plus DHA, compared to each drug alone (Figure 2). Our results support that osimertinib plus DHA represents an interesting and clinically applicable combination in HNSCC. 

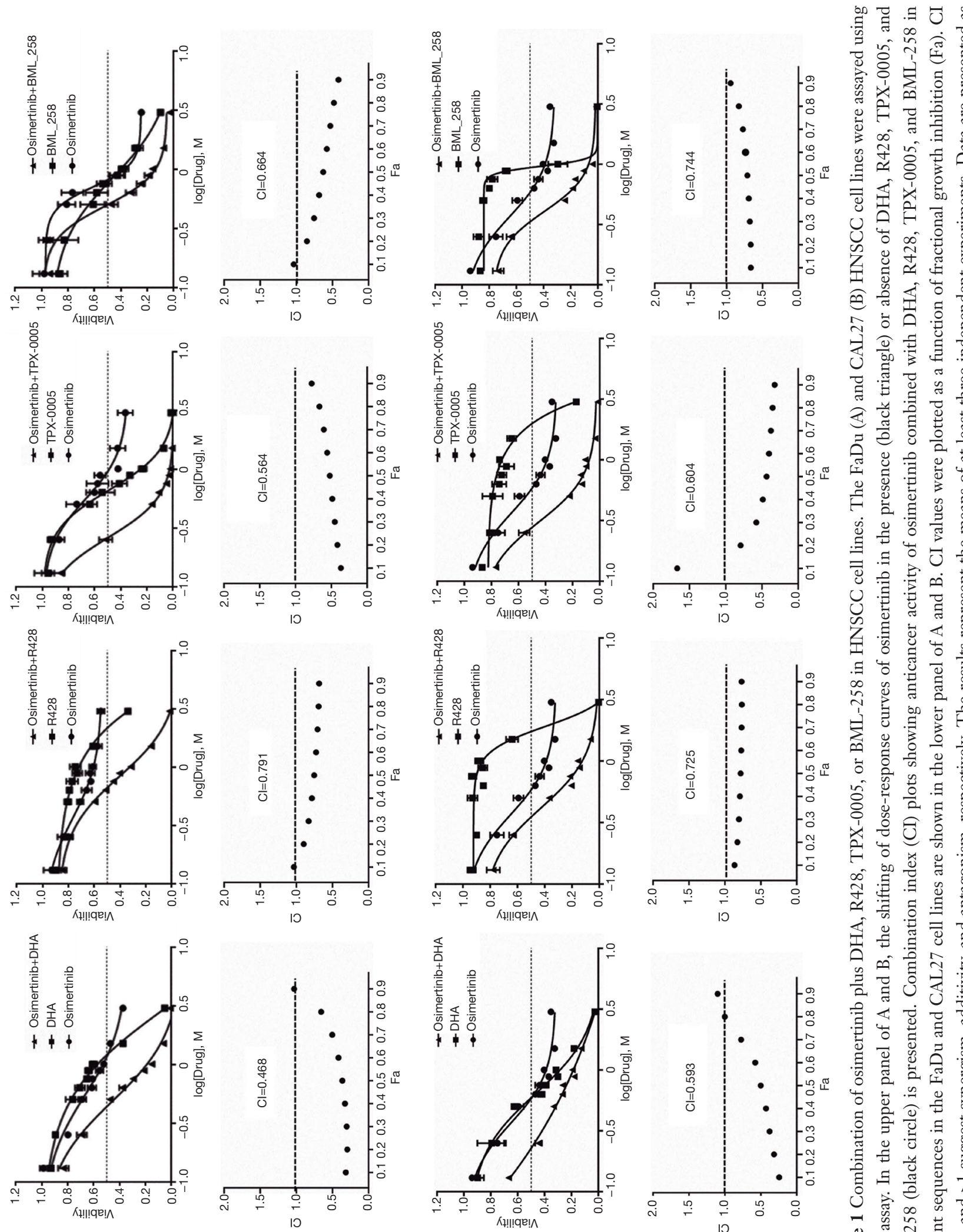

$\infty$

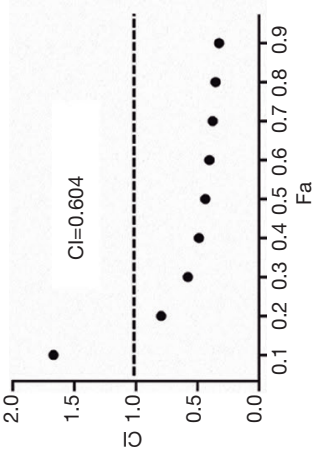

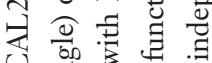

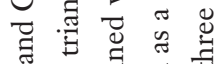

¿

సै

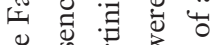

$\risingdotseq$ 芒.

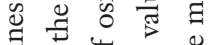

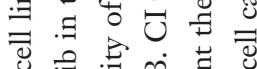

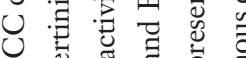

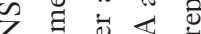

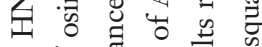

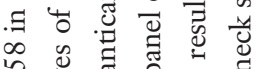

î

$\sum_{0} 0$

क्षे की च

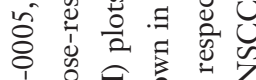

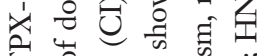

$\infty$ on

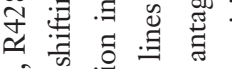

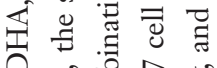

คी है ิિ

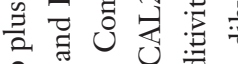

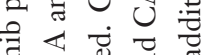

पै

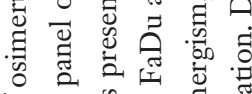

पे⿺辶寸

.

है

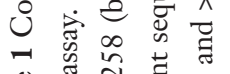

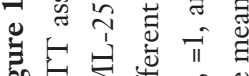

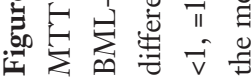



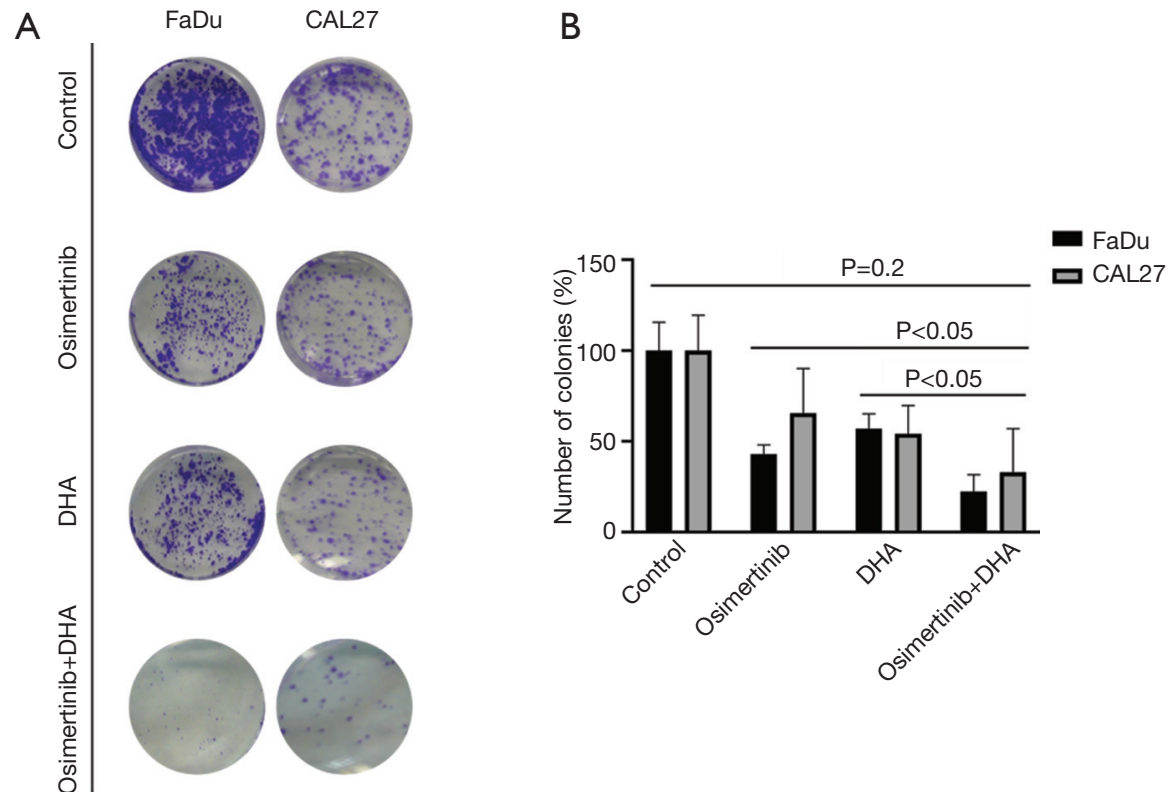

Figure 2 Colony formation assay upon treatment of HNSCC cell lines with osimertinib, DHA or the double combination. (A) FaDu and CAL27 cells were grown in six-well plates (1,000 cells/well) for 24 hours and then left untreated or treated with osimertinib, DHA and the double combination. After 72 hours, media was replaced with fresh media without drugs. After 7 more days cells were washed and stained with crystal violet and then photographed. The crystal violet was extracted and assayed by spectrophotometry. The absorbance was measured at $570 \mathrm{~nm}$. Osimertinib was used at $0.02 \mu \mathrm{M}$ and DHA at $20 \mu \mathrm{M}$. Images are representative of at least three independent experiments. (B) Quantification of the colonies treated with the indicated concentrations of osimertinib, DHA, and osimertinib plus DHA in A. Values are relative to those of dimethyl sulfoxide-treated (control) cells and represent the mean \pm standard deviation from three independent experiments. Statistical significance was assessed by one-tailed t-test. $\mathrm{P}$ value was calculated comparing all conditions (control, osimertinib alone, DHA alone) with osimertinib + DHA. DHA, dihydroartemisinin; HNSCC, head and neck squamous cell carcinoma.

\section{Correlation between signaling nodes and cellular responses to $\mathrm{DHA}$ or to osimertinib-DHA combination}

To further confirm the effect of the osimertinib plus DHA combination in HNSCC cells, western blotting and quantitative real-time PCR were performed to determine the gene expression on protein and mRNA level. As expected, DHA monotherapy almost completely abrogated the STAT3 phosphorylation on the critical tyrosine 705 (Tyr705) in a dose-dependent manner (Figure 3A). Surprisingly, we could see an inhibitory effect of DHA alone on the phosphorylation of other kinases related with resistance to EGFR inhibitors, like Src, paxillin, YAP1, and FAK. DHA repressed the phosphorylation of the transmembrane protein CUB domain-containing protein 1 (CDCP1) and the MET RTK. Some effect of DHA could also be seen on the AXL protein expression, especially in the CAL27 cell line (Figure $3 A$ ). Similarly to what we have reported in
EGFR-mutation positive NSCLC cells (4,5), osimertinib alone induced STAT3, Src, paxillin, YAP1, and FAK phosphorylation in the FaDu and CAL2 7 HNSCC cell lines (Figure 3B). DHA and the combination of DHA plus osimertinib reversed this phenomenon. The double combination was able to inhibit CDCP1 and MET phosphorylation, as well as AXL expression, more profoundly than each of the drugs alone (Figure $3 B$ ). Gene expression analysis demonstrated that after 7 days of therapy, osimertinib plus DHA significantly decreased AXL, CDCP1 and MET mRNA expression levels compared to those in FaDu and CAL27 cells at baseline and/or treated with osimertinib alone (Figure 4). Based on previous knowledge that DHA decreases the levels of glutathione peroxidase (GPX4) inducing ferroptoptic cell death in HNSCC (23), we added GPX4 in the transcripts that we explored. As shown in Figure 4, when osimertinib was combined with DHA, it could downregulate the expression levels of GPX4 in both HNSCC cell lines 
A

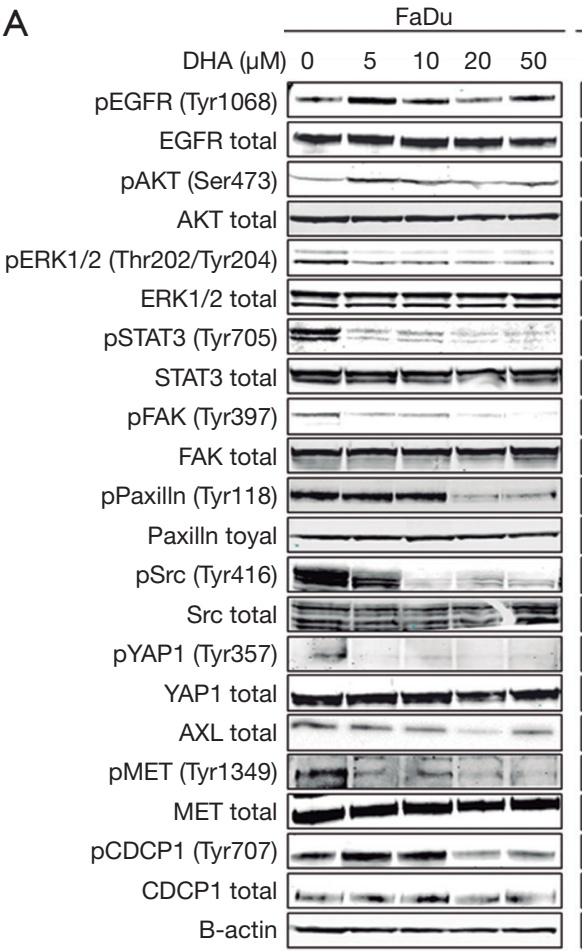

CAL27

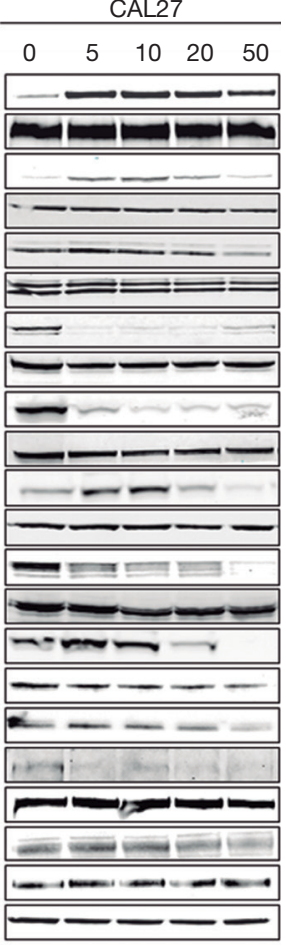

B

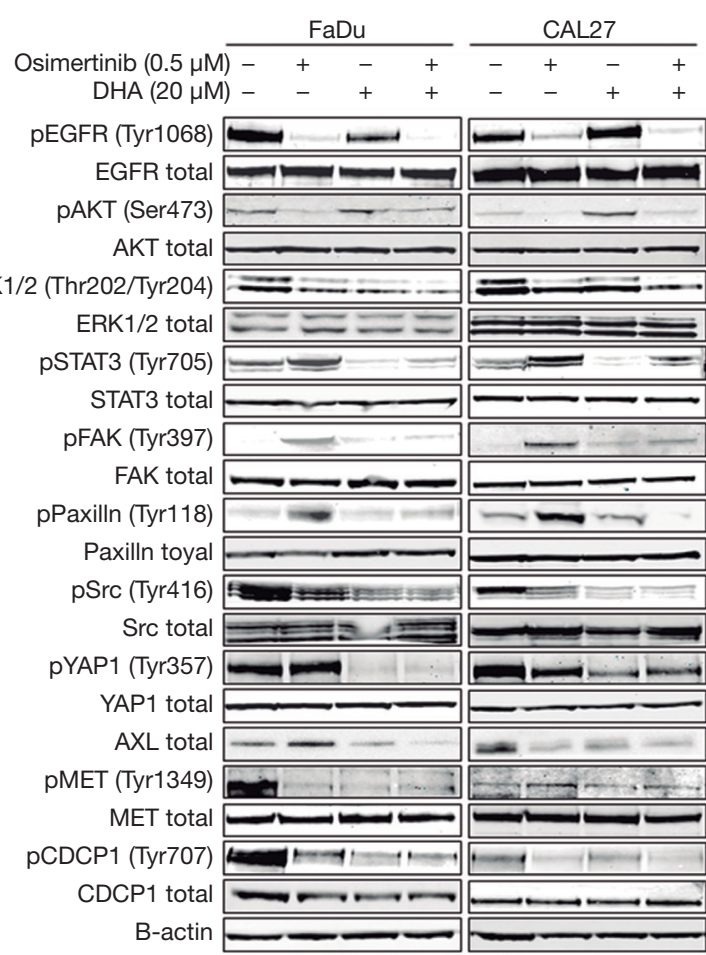

Figure 3 Effects of single treatments or osimertinib plus DHA combination on proteins involved in the downstream signaling of the EGFR pathway and RTKs. Western blot analysis showing protein levels in FaDu and CAL27 cells treated with DHA (A) and osimertinib plus DHA (B). In A, cells were treated with increasing doses of DHA for 24 hours. In B, cells were treated with the single drugs (0.5 $\mu M$ osimertinib, and $20 \mu \mathrm{M} \mathrm{DHA}$ ) or the combination and protein extracts were taken at $24 \mathrm{~h}$ after treatment. B-actin was used as loading control. Data were generated from a minimum of three replicates. DHA, dihydroartemisinin; EGFR, epidermal growth factor receptor; RTK, receptor tyrosine kinase.

tested, but the difference was not statistically significant.

The prognostic significance of AXL, CDCP1 and MET for HNSCC was confirmed in The Cancer Genome Atlas (TCGA) HNSCC gene expression dataset [(R2: Genomics Analysis and Visualization Platform (http://r2.amc.nl)]. In a dataset of 165 stage IV HNSCC specimens, high CDCP1 and MET expression were significantly related to shorter overall survival. High AXL mRNA expression was also related to shorter overall survival, although the difference was not statistically significant (Figure S2).

\section{Effect of osimertinib and DHA combinational treatment in vivo}

Our in vitro data prompted us to explore the effect of osimertinib plus DHA in vivo. Mice bearing $\mathrm{FaDu}$ and CAL27 xenografts were treated with $0.5 \%$ carboxymethyl cellulose sodium salt (vehicle), osimertinib $5 \mathrm{mg} / \mathrm{kg}$, DHA
$50 \mathrm{mg} / \mathrm{kg}$, or osimertinib plus DHA. The treatment with osimertinib plus DHA induced statistically significant tumor regression within 30 days of treatment. At this time point, mean (SD) tumor weights for vehicle, DHA, osimertinib, and osimertinib plus DHA, were 2.33 (0.83) g, $1.19(0.46) \mathrm{g}$, $0.76(0.26) \mathrm{g}$, and $0.49(0.18) \mathrm{g}$, respectively for $\mathrm{FaDu}$ xenografts, and $0.30(0.10) \mathrm{g}, 0.13(0.03) \mathrm{g}, 0.13(0.05) \mathrm{g}$, and $0.08(0.02) \mathrm{g}$, respectively for CAL27 xenografts. The adjusted $\mathrm{P}$ values of the differences between tumor weights of osimertinib versus osimertinib plus DHA, and DHA versus osimertinib plus DHA were both less than 0.05 , for $\mathrm{FaDu}$ and CAL27 xenografts (Figure 5). All treatment regimens were well tolerated as indicated by an absence of weight loss (Figure S3). These data confirmed our in vitro findings, demonstrating that tumors derived from representative HNSCC cell lines can be suppressed efficiently in vivo using the combination of osimertinib plus DHA without obvious side effects. 

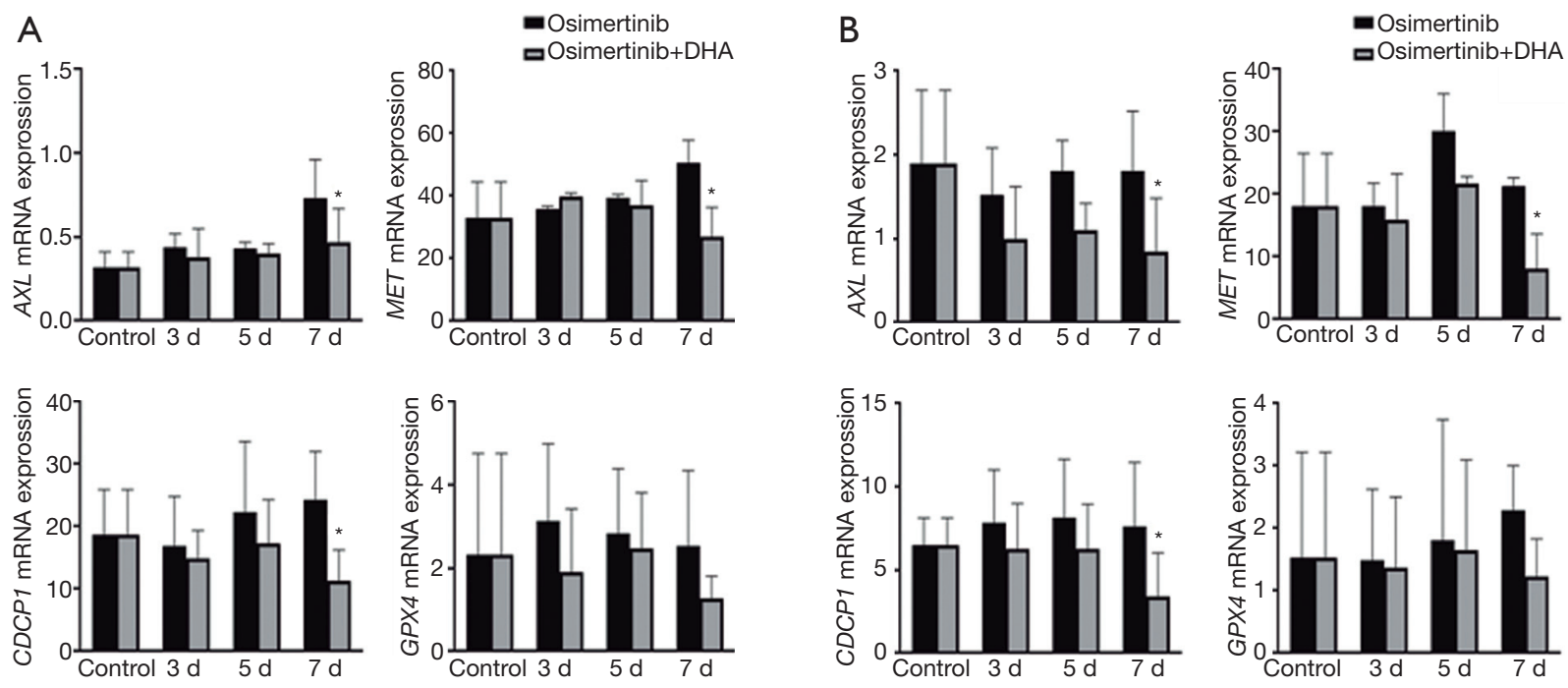

Figure 4 Effect of osimertinib and osimertinib plus DHA on the expression of AXL, MET, CDCP1, and GPX4. (A) FaDu and (B) CAL27 cells were treated with $0.5 \mu \mathrm{M}$ of osimertinib or $0.5 \mu \mathrm{M}$ of osimertinib plus $20 \mu \mathrm{M}$ of DHA for three, five and seven days. AXL, MET, CDCP1 and GPX4 mRNA expression were measured using quantitative reverse transcription-PCR. Data were generated from a minimum of three replicates. $\beta$-actin was used to normalize gene expression. Data are presented as the means \pm standard deviation. The statistical significance was assessed between cell lines treated with osimertinib + DHA and cell lines at basal conditions and treated with osimertinib alone; * two-sided $\mathrm{P}<0.05$. DHA, dihydroartemisinin.

\section{Discussion}

Recurrent or metastatic HNSCC is still treated with platinum, 5-fluoruracil and cetuximab [EXTREME trial, (1)], but with poor survival. Cetuximab alone shows a response rate of $13 \%$ with a median progression free survival of 2.3 months and an overall survival of 6 months. In addition, PD-1 inhibitors, such as nivolumab and pembrolizumab, induce discreet response in $13-15 \%$ of patients, also with a median free survival of 2 months, and a median overall survival of 7.5 to 8.4 months $(18,24)$. In the current study, we use a 4-tiered approach with the HNSCC cell lines, FaDu and CAL27, in order to retrieve information regarding combinatory therapy activity that could block important signaling pathways already reported in HNSCC. For example, the overexpression of AXL as a compensatory mechanism to cetuximab (8), SPHK1, which interacts with EGFR, augmenting downstream signaling (25), targeting STAT3, a common master transcriptional regulator, downstream of several RTKs, such as EGFR, AXL, MET and others (5), and, finally, blocking Src and
FAK, which could lead to downstream repression of YAP1 and block the feedback expression of RTKs, such as AXL and MET (4); (see Figure 6). The combination of the oral TKI osimertinib with SPHK1 inhibitor (BML 258), AXL inhibitor (R428), the multikinase inhibitor, repotrectinib (TPX-0005), and the STAT3 inhibitor, DHA, all show synergism. We chose to continue the experiments with the combination of osimertinib plus DHA, since DHA can be used as a repurposing drug (26). The combination of DHA and osimertinib was highly effective in vitro and in vivo in both $\mathrm{FaDu}$ and CAL27 cell lines. Moreover, the combination was able to shut down the expression of AXL, MET, CDCP1 and downstream main effectors, such as, STAT3, SRC and YAP1 (see Figures 3 and 6 ).

DHA is a semi-synthetic derivative of artemisinin, a first-line anti-malarial drug In Africa. DHA-piperaquine is used for the prevention of malaria in pregnant women (27). Artesunate and artemether are derivatives of artemisinin that are converted into their active metabolite DHA. Artesunate is approved for the treatment of uncomplicated and multidrug-resistant malaria and is currently in clinical trials 

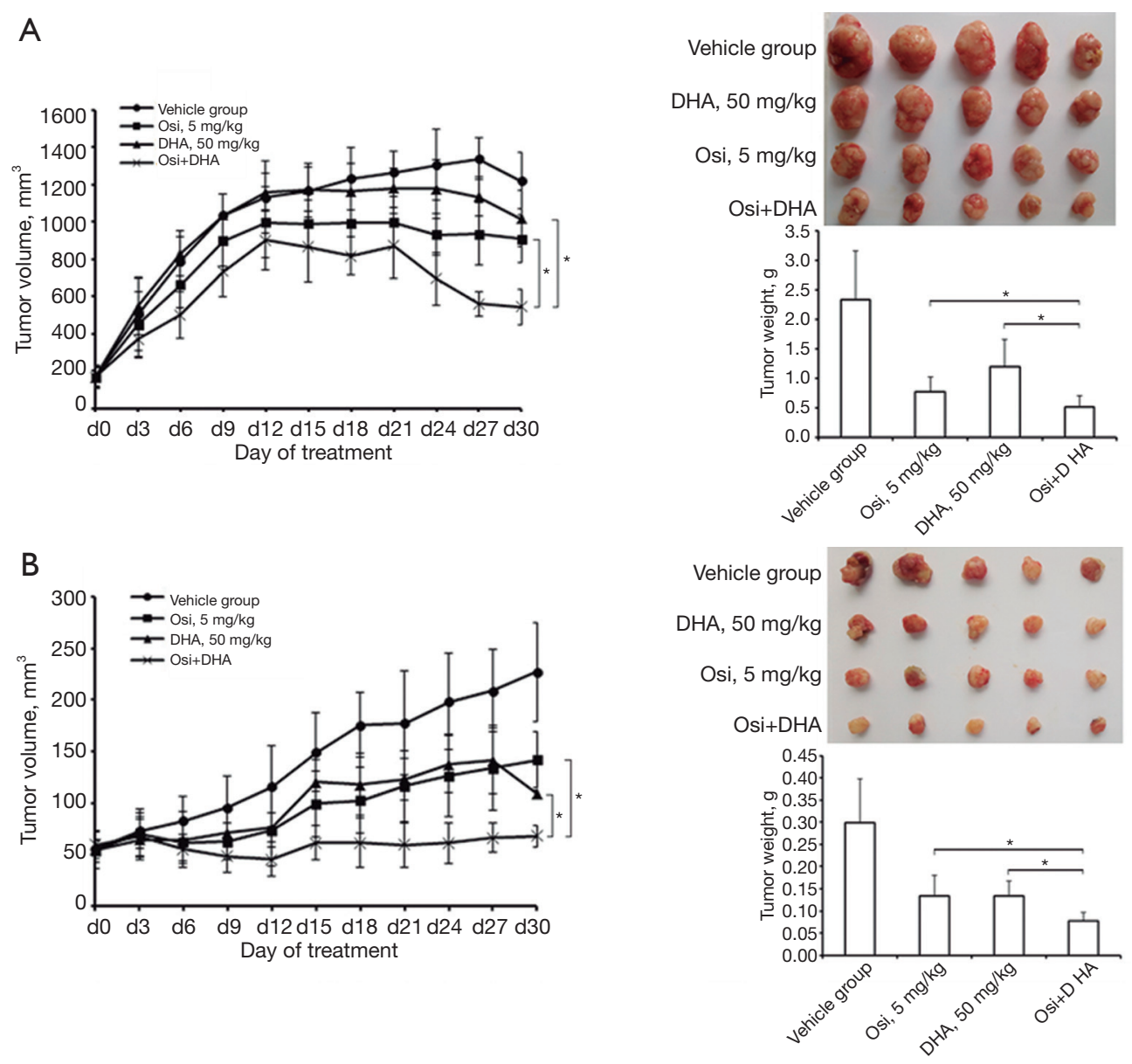

Figure 5 Anticancer effect of osimertinib plus DHA combination on FaDu and CAL27 xenografts in nude mice. Athymic nude female mice bearing $\mathrm{FaDu}(\mathrm{A})$ and CAL27 (B) xenografts were orally treated with $0.5 \%$ carboxymethyl cellulose sodium salt (vehicle), osimertinib $5 \mathrm{mg} / \mathrm{kg}$, DHA $50 \mathrm{mg} / \mathrm{kg}$, or osimertinib plus DHA. Tumor sizes were measured two or three times per week. Mean tumor volume and standard deviation are shown in A and B. Representative Photographs of mice in each group taken 30 days after treatment are shown in the upper panel of $\mathrm{C}(\mathrm{FaDu})$ and $\mathrm{D}$ (CAL27). The mean weight of tumors in nude mice at the time of sacrifice is presented in the lower panels of $\mathrm{C}$ (FaDu) and $\mathrm{D}$ (CAL27). Data shown are means with standard deviations of the tumor weight from five individual mice in each group. ${ }^{*} \mathrm{P}<0.05$. All $\mathrm{P}$ values were calculated by one-way ANOVA. DHA, dihydroartemisinin.

for colorectal (NCT02633098), breast (NCT00764036), and hepatocellular (NCT02304289) carcinoma.

Intriguingly, DHA decreases the levels of GPX4 in HNSCC, indicative of ferroptoptic cell death in HNSCC cancer cells (23). It has been shown that inducers of ferroptosis, such as erastin, a cystine-glutamate antiporter, or RSL3, a glutathione peroxidase inhibitor, can overcome cisplatin-resistance in HNSCC (28). Targeting GPX4 may represent a therapeutic strategy to prevent acquired resistance (29). Notwithstanding, nuclear factor (erythroidderived 2)-like 2 (NRF2) elevation could mediate resistance to DHA in HNSCC (30). A clinical study could be of utmost importance to confirm the benefit of DHA as a repurposing anti-cancer drug in combination with osimertinib for patients with recurrent or metastatic HNSCC. 


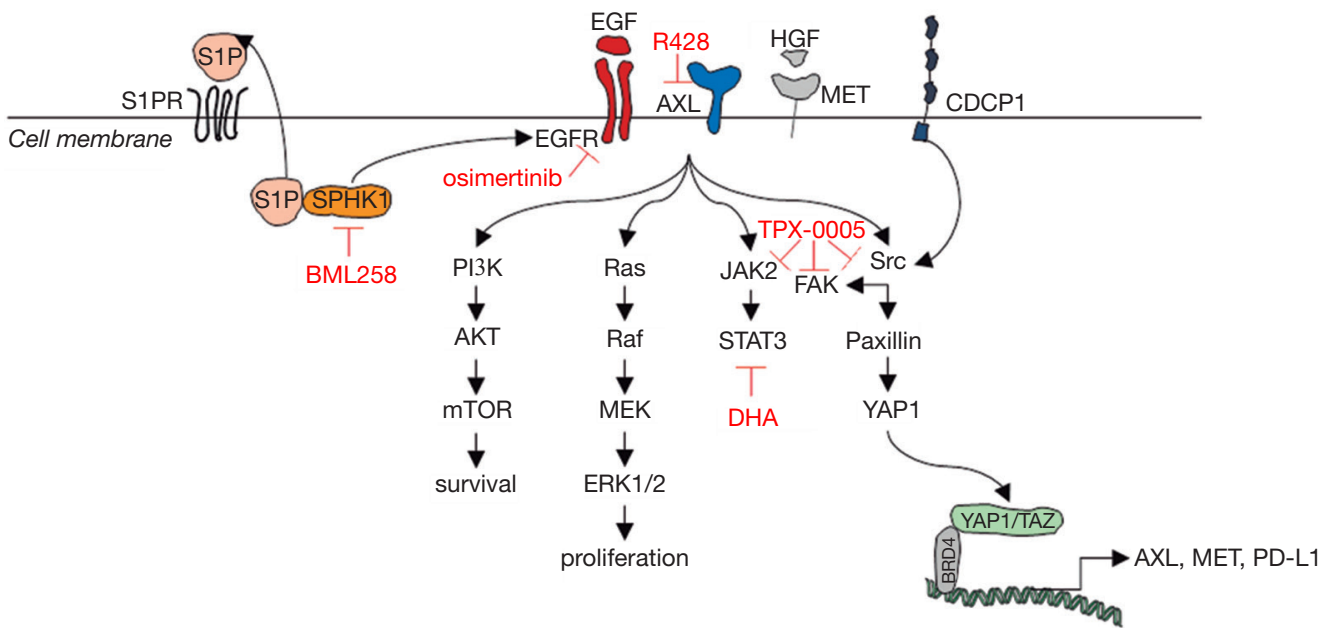

Figure 6 Our model in HNSCC. Activation of EGFR signaling in HNSCC occurs through multiple mechanisms, including overexpression of EGFR and its ligands, EGFR gene amplification or mutations, and EGFR transactivation by other receptor tyrosine kinases (RTKs) like AXL. Sphingosine-1-phosphate (S1P)/sphingosine kinase 1 (SPHK1) signaling can activate G-protein-coupled sphingosine 1-phosphate receptor (S1PRs) that subsequently transactivate EGFR. EGFR activation leads to the activation of mitogen-activated protein kinase (MAPK), signal transducer and activator of transcription 3 (STAT3) and AKT pathways. Raf activates MAPK/ERK Kinase 1 (MEK1) and MEK2. Extracellular signal-regulated kinase (ERK1 and ERK2) is activated upon phosphorylation by MEK1/2. Dual threonine 202 and tyrosine 204 residue phosphorylations activate ERK1. AKT is the major downstream target of phosphatidylinositol 3-kinase (PI3K), which is activated by EGFR. When PI3K is activated induces the recruitment of AKT to the cell membrane. The recruitment of AKT to the cell membrane drives a conformational change in the protein. This enables AKT phosphorylation on serine 473, which is required for full activation. Following activation, AKT translocates to the cytoplasm and nucleus, and phosphorylates various downstream substrates including mammalian target of rapamycin (mTOR). mTOR regulates the serine/threonine kinase, p70S6K, whose function regulates protein synthesis. STAT3 is activated by growth factor receptors, like EGFR, AXL or MET. Janus-like kinase 2 JAK2 mediates STAT3 phosphorylation and activation. When activated, STAT3 undergoes phosphorylation-induced homodimerization, leading to nuclear translocation, DNA binding and gene transcription. CUB domain-containing protein-1 (CDCP1) provides an alternative signaling mechanism for EGFR and is one of the main activators of Src signaling. Upon Src activation, several downstream Src binding partners are targeted for phosphorylation, including paxillin, YES associated protein 1 (YAP1), and focal adhesion kinase (FAK). As a transcriptional coactivator, YAP1 has been reported to bind several DNA-binding transcription factors. An interaction of YAP1/transcriptional coactivator with PDZ-binding motif (TAZ) with bromodomain-containing protein-4 (BRD4), leads to increased transcription of a large set of genes, including AXL, MET, and the immune checkpoint programmed death-ligand 1 (PD-L1). Osimertinib is an irreversible EGFR tyrosine kinase inhibitor, dihydroartemisinin (DHA) is a STAT3 inhibitor, R428 is an AXL inhibitor, TPX-0005 is a Src/FAK/JAK2 inhibitor, and BML258 is a SPHK1 inhibitor. EGFR, epidermal growth factor receptor; HNSCC, head and neck squamous cell carcinoma.

\section{Acknowledgments}

Funding: Work in Dr. Rosell's laboratory is partially supported by a grant from La Caixa Foundation, an Instituto de Salud Carlos III grant (RESPONSE, PIE16/00011), an Instituto de Salud Carlos III grant (PI14/01678), a Marie SkłodowskaCurie Innovative Training Networks European Grant (ELBA No. 765492) and a Spanish Association Against Cancer (AECC) grant (PROYE18012ROSE). Work in Dr. Cao's laboratory is partially supported by the Major National Science and Technology Program of China for Innovative Drug (2017ZX09101002-002-006), the Priority Academic Program Development of Jiangsu Higher Education Institutions (Integration of Chinese and Western Medicine) grant, Key R\&D Program of Jiangsu Province (BE2018755) and National Natural Science Foundation of China (No. 81573665).

\section{Footnote}

Conflicts of Interest: The authors have no conflicts of interest 
to declare.

Ethical Statement: The authors are accountable for all aspects of the work in ensuring that questions related to the accuracy or integrity of any part of the work are appropriately investigated and resolved. All procedures were based on Guide for Care and Use of Laboratory Animals of National Institutes of Health and approved by Institutional Animal Care and Use Committee of Jiangsu Province Academy of Traditional Chinese Medicine (SYXK 2016-0018).

\section{References}

1. Vermorken JB, Mesia R, Rivera F, et al. Platinum-based chemotherapy plus cetuximab in head and neck cancer. $\mathrm{N}$ Engl J Med 2008;359:1116-27.

2. Rubin Grandis J, Melhem MF, Gooding WE, et al. Levels of TGF-alpha and EGFR protein in head and neck squamous cell carcinoma and patient survival. J Natl Cancer Inst 1998;90:824-32.

3. Chung CH, Ely K, McGavran L, et al. Increased epidermal growth factor receptor gene copy number is associated with poor prognosis in head and neck squamous cell carcinomas. J Clin Oncol 2006;24:4170-6.

4. Karachaliou N, Chaib I, Cardona AF, et al. Common Co-activation of AXL and CDCP1 in EGFR-mutationpositive Non-small Cell Lung Cancer Associated With Poor Prognosis. EBioMedicine 2018;29:112-27.

5. Chaib I, Karachaliou N, Pilotto S, et al. Co-activation of STAT3 and YES-Associated Protein 1 (YAP1) Pathway in EGFR-Mutant NSCLC. J Natl Cancer Inst 2017. doi: 10.1093/jnci/djx014.

6. Jacobsen K, Bertran-Alamillo J, Molina MA, et al. Convergent Akt activation drives acquired EGFR inhibitor resistance in lung cancer. Nat Commun 2017;8:410.

7. Brand TM, Iida M, Stein AP, et al. AXL mediates resistance to cetuximab therapy. Cancer Res 2014;74:5152-64.

8. Giles KM, Kalinowski FC, Candy PA, et al. Axl mediates acquired resistance of head and neck cancer cells to the epidermal growth factor receptor inhibitor erlotinib. Mol Cancer Ther 2013;12:2541-58.

9. Balaji K, Vijayaraghavan S, Diao L, et al. AXL Inhibition Suppresses the DNA Damage Response and Sensitizes Cells to PARP Inhibition in Multiple Cancers. Mol Cancer Res 2017;15:45-58.

10. Elkabets M, Pazarentzos E, Juric D, et al. AXL mediates resistance to PI3Kalpha inhibition by activating the EGFR/PKC/mTOR axis in head and neck and esophageal squamous cell carcinomas. Cancer Cell 2015;27:533-46.

11. Soulieres D, Faivre S, Mesia R, et al. Buparlisib and paclitaxel in patients with platinum-pretreated recurrent or metastatic squamous cell carcinoma of the head and neck (BERIL-1): a randomised, double-blind, placebocontrolled phase 2 trial. Lancet Oncol 2017;18:323-35.

12. Baumann C, Ullrich A, Torka R. GAS6-expressing and self-sustaining cancer cells in $3 \mathrm{D}$ spheroids activate the PDK-RSK-mTOR pathway for survival and drug resistance. Mol Oncol 2017;11:1430-47.

13. Cross DA, Ashton SE, Ghiorghiu S, et al. AZD9291, an irreversible EGFR TKI, overcomes T790M-mediated resistance to EGFR inhibitors in lung cancer. Cancer Discov 2014;4:1046-61.

14. Chen Z, Chen Y, Xu M, et al. Osimertinib (AZD9291) Enhanced the Efficacy of Chemotherapeutic Agents in ABCB1- and ABCG2-Overexpressing Cells In Vitro, In Vivo, and Ex Vivo. Mol Cancer Ther 2016;15:1845-58.

15. Saenz-Ponce N, Pillay R, de Long LM, et al. Targeting the XPO1-dependent nuclear export of E2F7 reverses anthracycline resistance in head and neck squamous cell carcinomas. Sci Transl Med 2018. doi: 10.1126/ scitranslmed.aar7223.

16. Leonard B, Brand TM, O'Keefe RA, et al. BET Inhibition Overcomes Receptor Tyrosine KinaseMediated Cetuximab Resistance in HNSCC. Cancer Res 2018;78:4331-43.

17. Zanconato F, Battilana G, Forcato M, et al. Transcriptional addiction in cancer cells is mediated by YAP/TAZ through BRD4. Nat Med 2018;24:1599-610.

18. Ferris RL, Blumenschein G Jr, Fayette J, et al. Nivolumab for Recurrent Squamous-Cell Carcinoma of the Head and Neck. N Engl J Med 2016;375:1856-67.

19. Wong YK, Xu C, Kalesh KA, et al. Artemisinin as an anticancer drug: Recent advances in target profiling and mechanisms of action. Med Res Rev 2017;37:1492-517.

20. Jia L, Song Q, Zhou C, et al. Dihydroartemisinin as a Putative STAT3 Inhibitor, Suppresses the Growth of Head and Neck Squamous Cell Carcinoma by Targeting Jak2/ STAT3 Signaling. PLoS One 2016;11:e0147157.

21. Chou TC. Drug combination studies and their synergy quantification using the Chou-Talalay method. Cancer Res 2010;70:440-6.

22. Kalish LH, Kwong RA, Cole IE, et al. Deregulated cyclin D1 expression is associated with decreased efficacy of the selective epidermal growth factor receptor tyrosine 
kinase inhibitor gefitinib in head and neck squamous cell carcinoma cell lines. Clin Cancer Res 2004;10:7764-74.

23. Lin R, Zhang Z, Chen L, et al. Dihydroartemisinin (DHA) induces ferroptosis and causes cell cycle arrest in head and neck carcinoma cells. Cancer Lett 2016;381:165-75.

24. Cohen EEW, Soulieres D, Le Tourneau C, et al. Pembrolizumab versus methotrexate, docetaxel, or cetuximab for recurrent or metastatic head-and-neck squamous cell carcinoma (KEYNOTE-040): a randomised, open-label, phase 3 study. Lancet 2019;393:156-67.

25. Sheu JJ, Lee CC, Hua CH, et al. LRIG1 modulates aggressiveness of head and neck cancers by regulating EGFR-MAPK-SPHK1 signaling and extracellular matrix remodeling. Oncogene 2014;33:1375-84.

26. Krishna S, Ganapathi S, Ster IC, et al. A Randomised, Double Blind, Placebo-Controlled Pilot Study of Oral

Cite this article as: Chaib I, Cai X, Llige D, Santarpia M, Jantus-Lewintre E, Filipska M, Pedraz C, Cui J, Yang J, Miao J, Sun R, Bracht JWP, Ito M, Codony-Servat J, Karachaliou N, Aguilar A, Rosell R, Cao P. Osimertinib and dihydroartemisinin: a novel drug combination targeting head and neck squamous cell carcinoma. Ann Transl Med 2019;7(22):651. doi: 10.21037/ atm.2019.10.80
Artesunate Therapy for Colorectal Cancer. EBioMedicine 2014;2:82-90.

27. Kakuru A, Jagannathan P, Muhindo MK, et al. Dihydroartemisinin-Piperaquine for the Prevention of Malaria in Pregnancy. N Engl J Med 2016;374:928-39.

28. Roh JL, Kim EH, Jang HJ, et al. Induction of ferroptotic cell death for overcoming cisplatin resistance of head and neck cancer. Cancer Lett 2016;381:96-103.

29. Hangauer MJ, Viswanathan VS, Ryan MJ, et al. Drugtolerant persister cancer cells are vulnerable to GPX4 inhibition. Nature 2017;551:247-50.

30. Roh JL, Kim EH, Jang H, et al. Nrf2 inhibition reverses the resistance of cisplatin-resistant head and neck cancer cells to artesunate-induced ferroptosis. Redox Biol 2017;11:254-62. 


\section{Supplementary}

Table S1 FaDu and CAL27 cell lines were treated with serial dilutions of DHA, R428, TPX-0005 and BML258 for 72 h. Cell viability was assessed using the MTT assay and the IC50 values were calculated

\begin{tabular}{lcc}
\hline Inhibitor & FaDu IC50 $(\mu \mathrm{M})$ & CAL27 IC50 $(\mu \mathrm{M})$ \\
\hline DHA & $20 \pm 4.5$ & $15 \pm 5.25$ \\
R428 & $1 \pm 0.2$ & $1.5 \pm 0.2$ \\
TPX-0005 & $1.5 \pm 0.5$ & $3 \pm 0.5$ \\
BML258 & $15 \pm 3.75$ & $15 \pm 5$ \\
\hline
\end{tabular}

DHA, dihydroartemisinin.

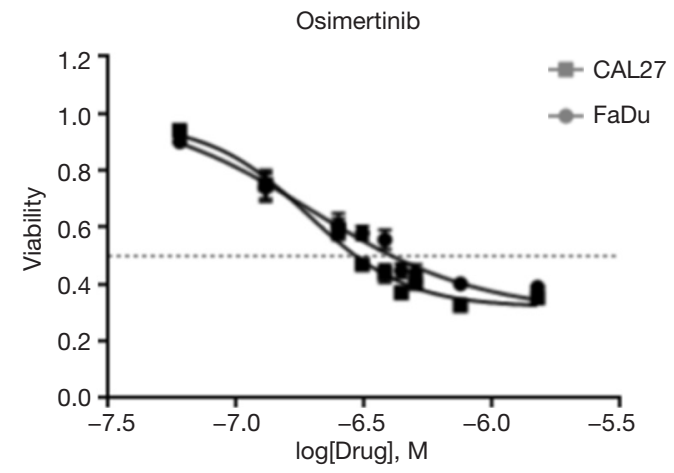

Figure S1 In vitro effect of osimertinib on proliferation of $\mathrm{FaDu}$ and CAL27 cell lines. MTT assays were used to examine the inhibitory activity on cell proliferation.
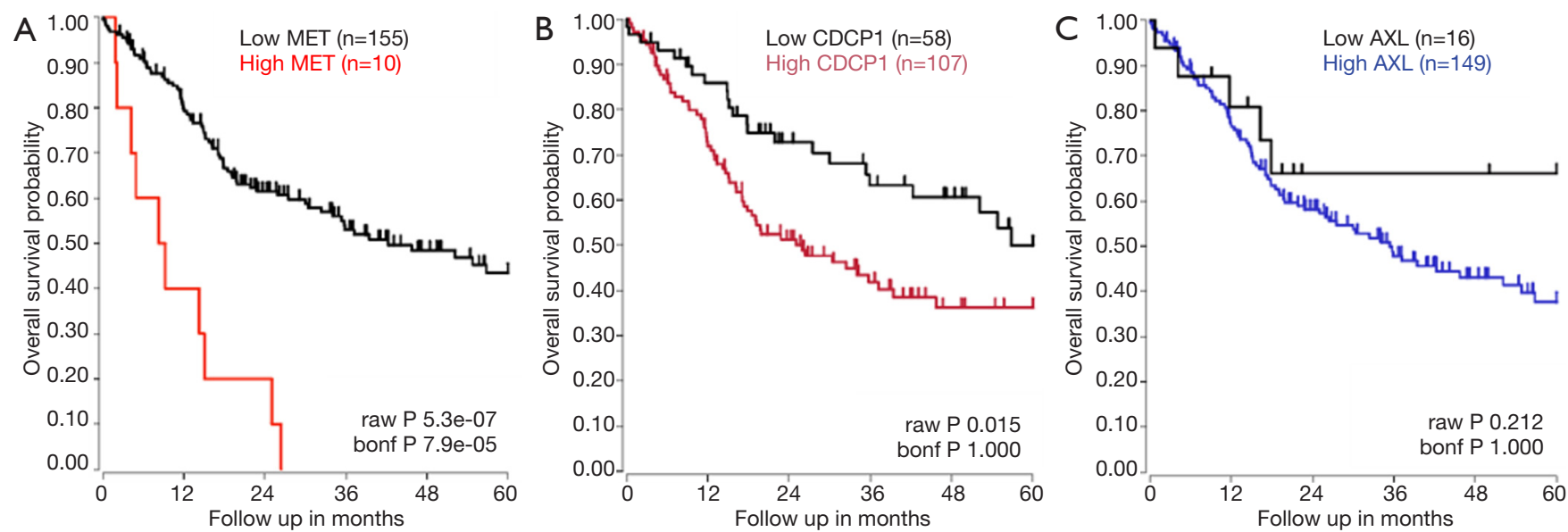

Figure S2 Correlation of MET, CDCP1 and AXL expression levels and survival in HNSCC patients. Kaplan-Meier survival plots showing that high MET (A), CDCP1 (B), and AXL (C) expression are associated with poor survival in 165 stage IV HNSCC patients in the TCGA dataset. HNSCC, head and neck squamous cell carcinoma; TCGA, The Cancer Genome Atlas.
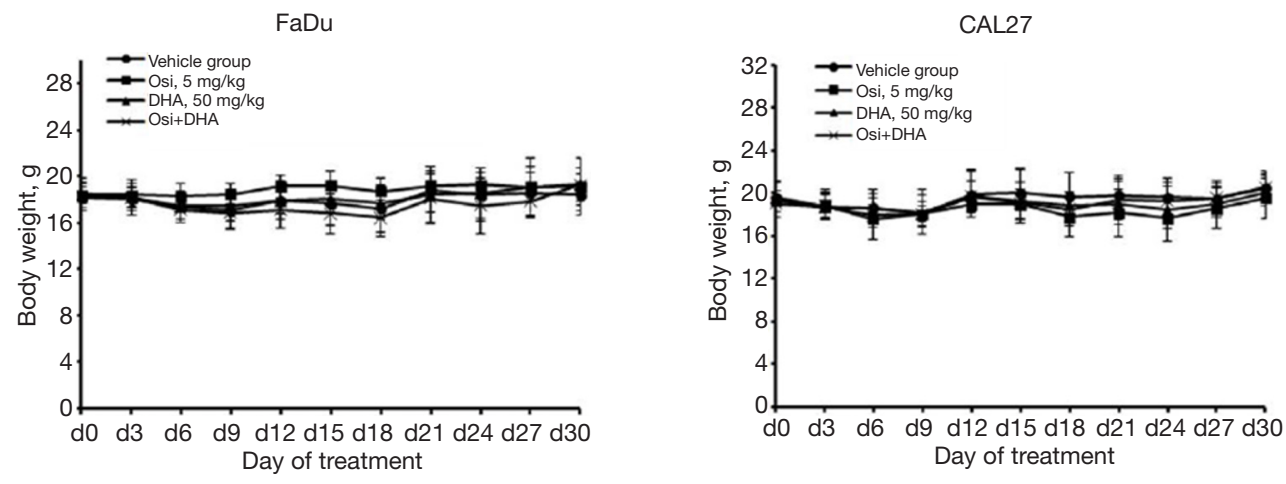

Figure S3 Mice's for each group (4 mice per group) were weighted during the course of treatments and mean values \pm SD were presented. 